OPEN ACCESS

Edited by:

Guo-Yuan Yang,

Shanghai Jiao Tong University, China

Reviewed by:

Daojun Hong

The First Affiliated Hospital of

Nanchang University, China

Fabio Pilato,

Policlinico Universitario Campus

Bio-Medico, Italy

${ }^{*}$ Correspondence:

Ming Liu

wyplmh@hotmail.com

Received: 11 November 2020

Accepted: 26 January 2021

Published: 19 February 2021

Citation:

Xu M, Li B, Zhong D, Cheng Y, Wu Q

Zhang S, Zhang S, Wu B and Liu M (2021) Cerebral Small Vessel Disease

Load Predicts Functional Outcome and Stroke Recurrence After Intracerebral Hemorrhage: A Median Follow-Up of 5 Years Front. Aging Neurosci. 13:628271 doi: 10.3389/fnagi.2021.628271

\section{Cerebral Small Vessel Disease Load Predicts Functional Outcome and Stroke Recurrence After Intracerebral Hemorrhage: A Median Follow-Up of 5 Years}

\author{
Mangmang $X u^{1}$, Baojin $L^{2}{ }^{2}$, Di Zhong ${ }^{1}$, Yajun Cheng ${ }^{1}$, Qian Wu ${ }^{1}$, Shuting Zhang ${ }^{1}$, \\ Shihong Zhang ${ }^{1}$, Bo Wu ${ }^{1}$ and Ming Liu ${ }^{1 *}$ \\ 'Department of Neurology, Center of Cerebrovascular Diseases, West China Hospital, Sichuan University, Chengdu, China, \\ ${ }^{2}$ Department of Rehabilitation Medicine, West China Hospital, Sichuan University, Chengdu, China
}

Background: Uncertainty exists over the long-term prognostic significance of cerebral small vessel disease (CSVD) in primary intracerebral hemorrhage $(\mathrm{ICH})$.

Methods: We performed a longitudinal analysis of CSVD and clinical outcomes in consecutive patients with primary ICH who had MRI. Baseline CSVD load (including white matter hyperintensities [WMH], cerebral microbleeds [CMBs], lacunes, and enlarged perivascular spaces [EPVS]) was evaluated. The cumulative CSVD score was calculated by combining the presence of each CSVD marker (range 0-4). We followed participants for poor functional outcome [modified Rankin scale $[\mathrm{mRS}] \geq 4$ ], stroke recurrence, and time-varying survival during a median follow-up of 4.9 [interquartile range [IQR] 3.1-6.0] years. Parsimonious and fuller multivariable logistic regression analysis and Cox-regression analysis were performed to estimate the association of CSVD markers, individually and collectively, with each outcome.

Results: A total of 153 patients were included in the analyses. CMBs $\geq 10$ [adjusted OR [adOR] 3.252, 95\% Cl 1.181-8.956, $p=0.023$ ] and periventricular $\mathrm{WMH}(\mathrm{PWMH})(\mathrm{adOR}$ $2.053,95 \% \mathrm{Cl} 1.220-3.456, p=0.007$ ) were significantly associated with poor functional outcome. PWMH (adOR 2.908, 95\% Cl 1.230-6.878, $p=0.015$ ) and lobar CMB severity (adOR 1.811, 95\% Cl 1.039-3.157, $p=0.036$ ) were associated with stroke recurrence. The cumulative CSVD score was associated with poor functional outcome (adOR 1.460, 95\% Cl 1.017-2.096) and stroke recurrence (adOR 2.258, 95\% Cl 1.080-4.723). Death occurred in $36.1 \%$ (13/36) of patients with CMBs $\geq 10$ compared with $18.8 \%(22 / 117)$ in those with $\mathrm{CMB}<10$ (adjusted HR 2.669, 95\% Cl 1.248-5.707, $p=0.011$ ). In addition, the cumulative CSVD score $\geq 2$ was associated with a decreased survival rate (adjusted HR 3.140, 95\% Cl 1.066-9.250, $p=0.038$ ).

Conclusions: Severe PWMH, CMB, or cumulative CSVD burden exert important influences on the long-term outcome of $\mathrm{ICH}$.

Keywords: cerebral small vessel disease, long-term follow-up, functional outcome, stroke recurrence, cerebral microbleeds 


\section{INTRODUCTION}

Spontaneous primary intracerebral hemorrhage (ICH) is mainly caused by cerebral small vessel disease (CSVD), including hypertensive arteriopathy (HA) and cerebral amyloid angiopathy (CAA) (Charidimou et al., 2016; Hankey, 2017), and has both short- and long-term consequences of high case fatality, disability, and risk of recurrent serious vascular events (Poon et al., 2014). Both HA and CAA are associated with magnetic resonance imaging (MRI) markers of CSVD, including lacunes, white matter hyperintensities (WMH), cerebral microbleeds (CMBs), and enlarged perivascular spaces (EPVS) (Charidimou et al., 2016). These MRI markers could reflect the presence and severity of CSVD-related damage (Wardlaw et al., 2013) and rarely occur in isolation. Thus, several semiquantitative scales have been developed to assess the cumulative CSVD burden (Staals et al., 2014; Xu et al., 2018).

Numerous studies have investigated the predictors of mortality and functional outcome following ICH (Gregório et al., 2018), whereas few have evaluated the role of CSVD in clinical outcomes after ICH (Sykora et al., 2017; Lioutas et al., 2018). At day 90, the cumulative CSVD burden and the presence of CMB (Lioutas et al., 2018), as well as severe WMH and increasing lacune number (Sykora et al., 2017), are important determinants of poor outcomes. Another study has shown that severe leukoaraiosis on CT scan is significantly associated with a poor functional outcome at discharge and with less recovery in mRS from discharge to day 90 (Uniken Venema et al., 2019).

However, to our knowledge, the effect of the CSVD burden on MRI on the long-term prognosis after ICH (for instance, beyond 1 year) has not been studied. Insights into the longitudinal association between baseline CSVD burden, individually and collectively, and long-term prognosis could possibly lead to a better appraisal of ICH and aid in the development of new therapeutic and preventative strategies to relieve the disease burden.

To address this gap, we aimed to investigate the association between individual CSVD markers and cumulative CSVD burden and (1) poor functional outcome, (2) stroke recurrence, and (3) time-varying survival.

\section{METHODS}

\section{Study Design}

We analyzed consecutive patients with primary ICH with MRI susceptibility-weighted imaging (MRI-SWI), admitted to either the Department of Neurosurgery or the Department of Neurology, West China Hospital, Sichuan University from

Abbreviations: CSVD, cerebral small vessel disease; ICH, intracerebral hemorrhage; WMH, white matter hyperintensities; $\mathrm{CMB}$, cerebral microbleed; HA, hypertensive arteriopathy; CAA, cerebral amyloid angiopathy; EPVS, enlarged perivascular spaces; IVH, intraventricular hemorrhage; GCS, Glasgow Coma Scale; DWMH, deep WMH; PWMH, periventricular WMH; BG, basal ganglia; CSO, centrum semiovale; SWI, susceptibility-weighted imaging; IQR, interquartile range; FLAIR, fluid-attenuated inversion recovery; adOR, adjusted OR; mRS, Modified Rankin Scale; ROC, receiver operating characteristic; AUC, area under the ROC curve.
January 2012 to October 2017 in a prospectively collected database. Our study was approved by the Ethics Committee on Biomedical Research, West China Hospital of Sichuan University. Informed consents were obtained from patients or their family members.

\section{Patient Selection}

A total of 203 consecutive patients with primary ICH with MRI-SWI examination in our ongoing prospective cohort study were screened. For our present analysis, patients were eligible if they (1) meet the diagnosis of primary ICH and (2) had MRI with adequate sequences at baseline for the assessments of lacunes, CMBs, WMH, and EPVS. Patients were excluded if (1) the hemorrhage was considered to be primary intraventricular hemorrhage (IVH), (2) the time of onset was beyond 3 months of symptom onset, (3) they had hemorrhagic transformation after an ischemic stroke, (4) their images were of poor quality, or (5) the time of onset was unclear. Angiography was carried out when clinical characteristics were suggestive of a vascular malformation.

\section{ICH Subtype Classification}

The ICH subtypes (HA-ICH, CAA-ICH, mixed-location ICH, and undetermined-ICH) were coded according to the previous studies (Linn et al., 2010; Pasi et al., 2018). We defined individuals as having HA-ICH when patients had deep ICH, with or without deep CMBs, but no lobar ICH or lobar CMBs. Those with strictly lobar ICH (cerebellar hemorrhage allowed) without deep ICH or CMBs were coded as having CAA-ICH when they aged $\geq 55$ years and as undetermined-ICH when they were younger than 55 years. ICH or CMBs in both deep and lobar regions were coded as mixed-location ICH.

\section{Data Collection}

We collected age, sex, vascular risk factors (including hypertension, diabetes mellitus, hyperlipidemia, cardiac disease, smoking, and alcohol consumption) (Valenti et al., 2016), a previous history of stroke, stroke severity [evaluated using Glasgow Coma Scale [GCS] score] on admission, serum factors (including blood glucose, albumin, cholesterol, highdensity lipoprotein, low-density lipoprotein, and creatinine), and treatment (surgical hematoma evacuation vs. conservative treatment) for each enrolled participant at baseline. We also collected 10 prespecified complications recorded by trained neurologists, which included pneumonia, urinary tract infection, gastrointestinal bleeding, seizure, septic shock, electrolyte disturbances, deep vein thrombosis, other infections, depression, and anxiety. A list of complications and their definitions are provided in Supplementary Table 1. The definition of each complication was the same as that in the previous studies (Langhorne et al., 2000; Indredavik et al., 2008; Dellinger et al., 2013; Li et al., 2019). We did not include chronic medical complications such as chronic chest infection, since they were beyond the scope of this study. 


\section{Imaging Acquisition and Analysis Computed Tomography}

Neurologists who were blinded to the clinical information of patients assessed the ICH location, the ICH volume, the presence of IVH, and midline shift. The ICH location was classified into lobar (hemorrhage originating at the cortex and subcortical junction), deep [hemorrhage located in the basal ganglia (BG), thalamus, or brain stem], or cerebellar ICH. We grouped cerebellar and brain stem ICH into infratentorial ICH. The $\mathrm{ICH}$ volume at baseline was assessed using the $\mathrm{ABC} / 2$ method (Kothari et al., 1996).

\section{Magnetic Resonance Imaging}

The median time from the onset to MRI examination was 6.4 [interquartile range [IQR] 3.9-15.8] days in our present study. All enrolled patients underwent MRI scanning, including T1- and T2-weighted, fluid-attenuated inversion recovery (FLAIR), and SWI sequences, with a magnetic field strength of $3 \mathrm{~T}$. The details of MRI parameters of the four sequences were described in our previous studies (Xu et al., 2018, 2019).

A lacune of presumed vascular origin was defined as a small lesion (3-20 $\mathrm{mm}$ in diameter) and CSF-like intensity with hyperintensities on T2-weighted and hypointensities on T1weighted images, with a perilesional halo on FLAIR images sometimes (Klarenbeek et al., 2013; Staals et al., 2014). WMH was assessed using the Fazekas scale, which was a simple scale rating separately for the deep and periventricular region on a 4-point scale ranging from 0 to 3 (Fazekas et al., 1987). We summed the deep WMH (DWMH) score and the periventricular WMH (PWMH) score to form a total WMH score (scored 06). The presence of $\mathrm{WMH}$ was defined as PWMH Fazekas 3 or/and DWMH Fazekas 2-3(Staals et al., 2014). A CMB lesion was defined as a small area of hypointense signal void on SWI sequences, round or ovoid, blooming, and at least partly surrounded by the brain parenchyma, in line with the current consensus criteria (Greenberg et al., 2009). We recorded the CMB number and their topographic distributions. The lobar CMB severity was defined as follows: $0=$ absence of lobar $\mathrm{CMB}, 1=$ 1-4 lobar CMBs, 2 = 5-9 lobar CMBs, 3 = 10-19 lobar CMBs, and $4=$ equal to or more than 20 lobar CMBs. Deep CMB severity was defined as follows: $0=$ absence of deep CMB, $1=$ 1-4 deep CMBs, 2 = 5-9 deep CMBs, $3=10-19$ deep CMBs, and $4=$ equal to or more than 20 deep CMBs. CMB mimics, such as calcium and iron deposits, were excluded using CT scans (Greenberg et al., 2009). EPVS were defined as fluid-filled spaces, linear, round, or ovoid, of $<3 \mathrm{~mm}$ in diameter and were evaluated on the axial T2-weighted images. The side of the slice with the highest EPVS number in BG and centrum semiovale (CSO) was recorded after reviewing all relevant slices for the two anatomical areas (Wardlaw et al., 2013).

The cumulative CSVD score was calculated using an ordinal 5point scale ranging from 0 to 4 by combining the four individual CSVD markers, with one point allocated to each of the following: the presence of any lacune, the presence of any $\mathrm{CMB}$, the presence of $\mathrm{WMH}$, and BG EPVS > 10 according to the previous studies (Staals et al., 2014; Song et al., 2017). We defined severe cumulative CSVD burden when the CSVD score was equal to or greater than the median value.

\section{Outcomes}

The primary outcome in this study was a poor functional outcome [the Modified Rankin Scale $[\mathrm{mRS}] \geq 4$, which means severe functional dependence or death] at the end of the followup. Prespecified second outcomes included stroke recurrence and time-varying survival. We followed patients with telephone interviews at 3 -months, 6-months, 1 -year, and $\geq 3$-years postICH. All patients were followed up from enrollment, until the occurrence of death, the last clinical documentation, or the end of the last follow-up (April 2020). A stroke recurrence (including either ischemic or hemorrhagic stroke) was confirmed by asking patients or their family members. We confirmed a stroke recurrence by asking if the patient went to medical institutions (both inpatient and outpatient settings) because of any episodes of new weakness or numbness in legs or/and arms, or new problems with speech or vision, with a new diagnosis of stroke (ischemic stroke or ICH) by specialists.

\section{Statistical Analysis}

Interrater agreement of the CSVD markers was good or excellent, as previously described (Xu et al., 2019). We used the Pearson's chi-squared test or the Fisher's exact test for categorical variables, and the Student $t$-test or the Mann-Whitney $U$ test for continuous variables, as appropriate. First, the association between risk factors and each outcome was estimated using the univariate analysis. Then, factors with $p<0.2$ were forced into multivariable analyses as covariates to determine the association of each individual CSVD marker and the cumulative CSVD score with clinical outcomes. We used multivariable logistic regression analysis to determine the association between CSVD and poor functional outcome and stroke recurrence and multivariable Cox proportional hazard regression analysis to assess the relationship of CSVD with time-varying survival. Potential risk factors, which were entered into the multivariable analysis of the association between CSVD and poor functional outcome, included age (continuous variable), sex, hypertension, cardiac disease, the GCS score (continuous variable), serum albumin (continuous variable), and any complication. In addition, we adjusted for IVH and infratentorial ICH further since the two were the commonly used factors of ICH prognosis in a recent meta-analysis (Gregório et al., 2018). In the multivariable analysis of the association of CSVD with stroke recurrence, we only included patients who were first-ever ICH and had data of stroke recurrence, adjusting for age, sex, and ICH subtypes. We used the receiver operating characteristic (ROC) curve and the area under the ROC curve (AUC) value for each MRI maker and the cumulative CSVD score to characterize the discrimination for poor functional outcome and stroke recurrence. DeLong et al. test was used to evaluate the differences between two AUC values for significance (DeLong et al., 1988). IBM SPSS Statistics (version 21) and MedCalc (version 19.1) were used for statistical analyses. $p<0.05$ was considered to be significant. 


\section{RESULTS}

A total of 203 patients with primary ICH and MRI-SWI examination were screened, and 35 out of 203 (17.2\%) patients were excluded for various reasons (see Figure 1). Thus, 168 $(82.8 \% ; 61.0 \pm 12.2$ years, age range $=36-88$ years $)$ patients were enrolled in our present study. During a median follow-up of 4.9 (IQR 3.1-6.0) years, 15 participants did not complete any follow-up, leaving 153 patients who were included in the analyses (Figure 1). Of these, 111 (72.5\%) were men; the most common ICH subtype was mixed-location ICH (43.1\%), followed by HAICH (39.9\%), and CAA-ICH (15.0\%). Three patients younger than 55 years with strictly lobar (or cerebellar) $\mathrm{ICH}$ and without deep CMB were classified into the undetermined subgroup.

Comparing with those who were lost to follow-up, completers had a lower GCS score $(p=0.029)$ and a lower rate of diabetes mellitus $(p=0.025)$. Other clinical characteristics, serum factors, individual CSVD marker, and the cumulative CSVD score were comparable between the two groups (see Supplementary Table 2).

\section{Primary Outcome}

\section{Association Between CSVD and Poor Functional Outcome}

At the end of follow-up, 30.1\% (46/153) developed poor functional outcome, older $(p<0.001)$, having lower GCS score $(p=0.004)$, and lower serum albumin levels $(p=0.001)$, as well as higher prevalence of any complication $(p=0.001)$ than those who did not develop poor functional outcome. The overall characteristics of the 153 patients in the poor functional outcome analysis were shown in Supplementary Table 3.

The univariate analysis showed that patients with poor functional outcome had a greater proportion of any lacune,

\section{All primary ICH patients with MRI-SWI admitted} between January 2012 and October $2017(\mathrm{~N}=203)$

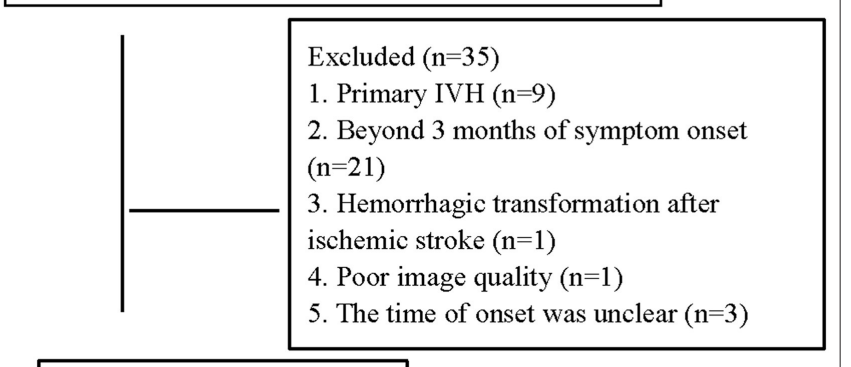

Patients enrolled $(\mathrm{n}=168)$

Did not complete any follow-up $(n=15)$

Final study sample $(\mathrm{n}=153)$

FIGURE 1 | Flow chart of patient selection. more severe PWMH, DWMH, and total WMH score, as well as a greater proportion of $\mathrm{CMB} \geq 10$ and $\mathrm{BG}$ EPVS $>20$, and expectedly higher cumulative CSVD score (all $p<0.05$ ) compared with those without a poor functional outcome (see Table 1).

After adjusting for the prespecified confounders higher PWMH score [adjusted OR[adOR] 2.085, 95\% CI 1.255-3.462, $p=0.005]$, higher total WMH score (adOR 1.395, 95\% CI 1.071$1.819, p=0.014), \mathrm{CMB} \geq 5($ adOR $2.885,95 \%$ CI $1.162-7.163$, $p=0.022), \mathrm{CMB} \geq 10$ (adOR 3.249, 95\% CI 1.201-8.794, $p=$ 0.020 ), and higher cumulative CSVD score (adOR 1.460, 95\% CI 1.017-2.096, $p=0.040$ ) were all significantly associated with poor functional outcome. The unfavorable prognostic effect of high CMB burden, PWMH severity on poor functional outcome was retained after adjusting for IVH and infratentorial ICH additionally (adOR 2.053, 95\% CI 1.220-3.456, $p=0.007$ for PWMH score; adOR 3.252, 95\% CI 1.181-8.956, $p=0.023$ for CMBs $\geq 10$ ) (see Table 2).

Then, we combined CMB $\geq 10$ ( $0=$ absence, $1=$ presence $)$ and PWMH score (Fazekas scale ranging 0-3) into a single score. With every point increase in this score, the risk of poor functional outcome increased by 2.1 times (adOR 95\% CI 1.327$3.352, p=0.002$ ) (Table 2). The predictive value of $\mathrm{CMB} \geq 10+$ PWMH for poor functional outcome performed better than the individual marker of $\mathrm{CMB} \geq 10(\mathrm{CMB} \geq 10+\mathrm{PWMH}$ vs. $\mathrm{CMB}$ $\geq 10$, AUC 0.704 vs. $0.580, p=0.003)$ or $\mathrm{CMB} \geq 5(\mathrm{CMB} \geq$ $10+\mathrm{PWMH}$ vs. $\mathrm{CMB} \geq 5$, AUC 0.704 vs. $0.582, p=0.007)$ (Supplementary Table 4).

\section{Secondary Outcomes}

\section{Association Between Cerebral Small Vessel Disease and Stroke Recurrence}

Of the 153 patients with mRS data, 15 had a previous history of stroke, and seven had missing data on recurrence, leaving 131 first-ever ICH patients who were included in the stroke recurrence analysis. During a median follow-up of 4.9 years, 14 patients experienced recurrent stroke (16 recurrent events overall; seven ICHs, six ischemic strokes, and three unclear stroke subtypes). The recurrence rate in $\mathrm{HA}-\mathrm{ICH}, \mathrm{CAA}-\mathrm{ICH}$, and mixed-location $\mathrm{ICH}$ was $3.5 \%(2 / 57), 5.6 \%(1 / 18)$, and $17.0 \%$ (9/53), respectively. The risk of stroke recurrence varied by ICH subtypes ( $p=0.006$, Fisher's exact test).

Compared to patients without stroke recurrence, recurrent patients were more likely to present with lacunes, WMH, lobar $\mathrm{CMB}$, and a higher cumulative CSVD score in the univariate analysis (all $p<0.05$ ) (Table 1). After adjusting for age, sex, and $\mathrm{ICH}$ subtypes, the unfavorable prognostic effect of PWMH (adOR 2.908, 95\% CI 1.230-6.878; $p=0.015)$, total WMH (adOR $1.602,95 \%$ CI $1.046-2.455 ; p=0.030$ ), lobar CMB severity (adOR $1.811,95 \%$ CI $1.039-3.157 ; p=0.036$ ), and the cumulative CSVD score (adOR 2.258, 95\% CI 1.080-4.723; $p=0.030$ ) on stroke recurrence was retained.

Similarly, we combined PWMH (Fazekas scale ranging from 0 to 3 ) and lobar CMB severity into a single score. The association between this score and stroke recurrence was substantially significant (adOR 1.847, 95\% CI 1.194-2.858, $p=0.006$ ) (Table 3). The predictive value of the combined CSVD burden 
TABLE 1 | Comparison between patients with and without poor functional outcome and between those with and without stroke recurrence after ICH.

\begin{tabular}{|c|c|c|c|c|c|c|}
\hline \multirow[t]{2}{*}{ Variables } & \multicolumn{3}{|c|}{ Poor functional outcome $(n=153)$} & \multicolumn{3}{|c|}{ Stroke recurrence $(n=131)$} \\
\hline & $\begin{array}{c}\text { No } \\
(n=107)\end{array}$ & $\begin{array}{c}\text { Yes } \\
(n=46)\end{array}$ & $p$ & $\begin{array}{c}\text { No } \\
(n=117)\end{array}$ & $\begin{array}{c}\text { Yes } \\
(n=14)\end{array}$ & $p$ \\
\hline Male, $n(\%)$ & $73(68.2)$ & 38 (82.6) & 0.068 & $88(75.2)$ & $10(71.4)$ & 0.750 \\
\hline \multicolumn{7}{|l|}{ ICH SUBTYPES, $\boldsymbol{n}(\%)$} \\
\hline $\mathrm{HA}-\mathrm{ICH}$ & $46(43.0)$ & $15(32.6)$ & 0.387 & $53(45.3)$ & $2(14.3)$ & 0.006 \\
\hline Undetermined & $3(2.8)$ & 0 & & $1(0.9)$ & $2(14.3)$ & \\
\hline \multicolumn{7}{|l|}{ ICH CHARACTERISTICS } \\
\hline Infratentorial ICH, $n(\%)$ & $16(15.0)$ & $8(17.4)$ & 0.704 & $17(14.5)$ & $4(28.6)$ & 0.239 \\
\hline GCS score*, mean \pm SD & $13.7 \pm 2.3$ & $12.2 \pm 3.0$ & 0.004 & $13.3 \pm 2.6$ & $12.8 \pm 3.1$ & 0.479 \\
\hline Hematoma volume ${ }^{\dagger}$, median (IQR) & $9.4(3.0-19.7)$ & $10.0(3.7-24.3)$ & 0.977 & $10.0(4.2-18.8)$ & $10.1(3.6-24.2)$ & 0.960 \\
\hline Systolic BP, mean \pm SD & $156.8 \pm 28.0$ & $159.3 \pm 29.5$ & 0.622 & $159.4 \pm 29.4$ & $159.9 \pm 24.0$ & 0.949 \\
\hline Diastolic BP, mean \pm SD & $93.8 \pm 15.9$ & $90.3 \pm 15.4$ & 0.209 & $93.8 \pm 16.7$ & $89.6 \pm 12.2$ & 0.372 \\
\hline Hypertension, $n(\%)$ & 89 (83.2) & $34(73.9)$ & 0.186 & $95(81.2)$ & $13(92.9)$ & 0.462 \\
\hline Diabetes mellitus, $n(\%)$ & $10(9.3)$ & $6(13.0)$ & 0.567 & $13(11.1)$ & $1(7.1)$ & 1.000 \\
\hline Hyperlipidemia, $n$ (\%) & $7(6.5)$ & $1(2.2)$ & 0.436 & $6(5.1)$ & 0 & 1.000 \\
\hline Prior stroke, $n(\%)$ & $12(11.2)$ & $3(6.5)$ & 0.555 & NA & NA & \\
\hline Smoking, $n(\%)$ & $30(28.0)$ & $16(34.8)$ & 0.404 & $36(30.8)$ & $5(35.7)$ & 0.763 \\
\hline Alcohol consumption, $n(\%)$ & $20(18.7)$ & $8(17.4)$ & 0.849 & $22(18.8)$ & $2(14.3)$ & 1.000 \\
\hline Cardiac disease, $n(\%)$ & $4(3.7)$ & $6(13.0)$ & 0.067 & $7(6.0)$ & $1(7.1)$ & 1.000 \\
\hline \multicolumn{7}{|l|}{ LABORATORY TEST, MEAN \pm SD } \\
\hline Blood glucose§ & $6.9 \pm 2.1$ & $7.0 \pm 2.4$ & 0.867 & $7.0 \pm 2.3$ & $6.9 \pm 1.7$ & 0.783 \\
\hline Albumin $\S$ & $42.5 \pm 4.0$ & $39.9 \pm 4.8$ & 0.001 & $41.9 \pm 4.4$ & $42.6 \pm 3.1$ & 0.598 \\
\hline Lacune $\geq 1$ & 42 (39.3) & $27(58.7)$ & 0.027 & $47(40.2)$ & $10(71.4)$ & 0.026 \\
\hline Lacune $\geq 2$ & $27(25.2)$ & $10(21.7)$ & 0.643 & $25(21.4)$ & $6(42.9)$ & 0.096 \\
\hline Lacune number & $0(0-2)$ & $1(0-1)$ & 0.122 & $0(0-1)$ & $1(0-2.5)$ & 0.023 \\
\hline The presence of WMH & $42(39.3)$ & $31(67.4)$ & 0.001 & 49 (41.9) & $11(78.6)$ & 0.009 \\
\hline PWMH score & $1(1-2)$ & $3(1-3)$ & $<0.001$ & $1(1-2)$ & $3(1.8-3)$ & 0.002 \\
\hline DWMH score & $1(1-2)$ & $2(1-3)$ & 0.003 & $1(1-2)$ & $2(0.8-3)$ & 0.048 \\
\hline Total WMH score & $2(2-4)$ & $4(2.8-6)$ & $<0.001$ & $2(2-4)$ & $5(3.3-6)$ & 0.011 \\
\hline The presence of $\mathrm{CMB}$ & $75(70.1)$ & 38 (82.6) & 0.106 & 83 (70.9) & $12(85.7)$ & 0.348 \\
\hline $\mathrm{CMBs} \geq 5$ & 36 (33.6) & $23(50.0)$ & 0.057 & $42(35.9)$ & $6(42.9)$ & 0.610 \\
\hline $\mathrm{CMBs} \geq 10$ & $20(18.7)$ & $16(34.8)$ & 0.031 & $24(20.5)$ & $6(42.9)$ & 0.088 \\
\hline Lober CMB number & $0(0-2)$ & $1(0-3.3)$ & 0.156 & $0(0-2)$ & $2(0-18.8)$ & 0.012 \\
\hline BG EPVS > 10 & $51(47.7)$ & $26(56.5)$ & 0.315 & $57(48.7)$ & $9(64.3)$ & 0.271 \\
\hline BG EPVS > 20 & $19(17.8)$ & $17(37.0)$ & 0.010 & $24(20.5)$ & $5(35.7)$ & 0.303 \\
\hline CSO EPVS > 20 & $34(31.8)$ & $14(30.4)$ & 0.870 & 35 (29.9) & $3(21.4)$ & 0.756 \\
\hline Cumulative CSVD score & $2(1-3)$ & $3(2-4)$ & 0.002 & $2(1-3)$ & $3(2-4)$ & 0.006 \\
\hline
\end{tabular}

ICH, intracerebral hemorrhage; HA, hypertensive arteriopathy; CAA, cerebral amyloid angiopathy; GCS, Glasgow Coma Scale; BP, blood pressure; SD, standard deviation; IQR, interquartile range; $I N H$, intraventricular hemorrhage; $H D L$, high-density lipoprotein; $L D L$, low-density lipoprotein; CSVD, cerebral small vessel disease; WMH, white matter hyperintensities; PWMH, periventricular WMH; DWMH, deep WMH; EPVS, enlarged perivascular spaces; BG, basal ganglia; CSO, centrum semiovale; CMB, cerebral microbleed.

*152 patients had data of the GCS score in the analysis of poor functional outcome and 130 in the analysis of stroke recurrence.

+119 patients had data of hematoma volume in the analysis of poor functional outcome and 106 in the analysis of stroke recurrence.

${ }^{\ddagger} 150$ patients had data of cholesterol, HDL, and LDL in the analysis of poor functional outcome and 129 patients had those data in the analysis of stroke recurrence.

\$ 152 patients had data of blood glucose, albumin, and creatinine in the analysis of poor functional outcome. 
TABLE 2 | Association of CSVD with poor functional outcome in the multivariable logistic regression analysis.

\begin{tabular}{|c|c|c|c|c|c|c|c|c|}
\hline & \multicolumn{4}{|c|}{ Mode I } & \multicolumn{4}{|c|}{ Model 2} \\
\hline & $p$ & OR & Lower & Upper & $p$ & OR & Lower & Upper \\
\hline \multicolumn{9}{|c|}{ INDIVIDUAL CSVD Marker } \\
\hline Lacune $\geq 1$ & 0.078 & 2.164 & 0.918 & 5.100 & 0.081 & 2.159 & 0.908 & 5.132 \\
\hline DWMH score & 0.092 & 1.505 & 0.936 & 2.420 & 0.134 & 1.446 & 0.892 & 2.345 \\
\hline Total WMH score & 0.014 & 1.395 & 1.071 & 1.819 & 0.022 & 1.373 & 1.047 & 1.801 \\
\hline $\mathrm{CMB} \geq 1$ & 0.056 & 2.884 & 0.972 & 8.554 & 0.074 & 2.722 & 0.908 & 8.162 \\
\hline $\mathrm{CMB} \geq 5$ & 0.022 & 2.885 & 1.162 & 7.163 & 0.020 & 2.984 & 1.187 & 7.500 \\
\hline $\mathrm{CMB} \geq 10$ & 0.020 & 3.249 & 1.201 & 8.794 & 0.023 & 3.252 & 1.181 & 8.956 \\
\hline
\end{tabular}

Model 1: Adjusted for age per year, sex, hypertension, cardiac disease, GCS per unit, serum albumin per unit, and any complication for each individual CSVD marker and each CSVD score.

Model 2: Adjusted for model $1+$ IVH and infratentorial ICH.

Assignment:

Cumulative CSVD score: one point allocated to each of the following markers: the presence of lacune, CMB, and the presence of WMH and BG EPVS > 10.

$C M B \geq 10+P W M H: C M B \geq 10(0=$ absence, $1=$ presence), $P W M H$ score (Fazekas scale ranging from 0 to 3 ).

TABLE 3 | Association of CSVD with stroke recurrence in multivariable logistic-regression.

\begin{tabular}{|c|c|c|c|c|c|c|c|c|}
\hline & \multicolumn{4}{|c|}{ Age + sex adjusted } & \multicolumn{4}{|c|}{ Age + sex $+\mathrm{ICH}$ subtypes adjusted } \\
\hline & $p$ & OR & Lower & Upper & $p$ & OR & Lower & Upper \\
\hline \multicolumn{9}{|l|}{ INDIVIDUAL CSVD Marker } \\
\hline The presence of lacune & 0.039 & 3.663 & 1.068 & 12.558 & 0.116 & 3.168 & 0.753 & 13.323 \\
\hline PWMH score & 0.003 & 3.127 & 1.457 & 6.711 & 0.015 & 2.908 & 1.230 & 6.878 \\
\hline DWMH score & 0.054 & 1.889 & 0.988 & 3.611 & 0.151 & 1.714 & 0.822 & 3.573 \\
\hline Total WMH score & 0.008 & 1.672 & 1.146 & 2.439 & 0.030 & 1.602 & 1.046 & 2.455 \\
\hline The presence of $\mathrm{CMB}$ & 0.276 & 2.386 & 0.500 & 11.396 & 0.416 & 2.615 & 0.258 & 26.506 \\
\hline Lobar CMB severity & 0.009 & 1.760 & 1.153 & 2.687 & 0.036 & 1.811 & 1.039 & 3.157 \\
\hline
\end{tabular}

Assignment of deep CMB severity: $0=$ absence of deep CMB, 1 = 1-4 deep CMBs, $2=5-9$ deep CMBs, 3 = 10-19 deep CMBs, $4=$ equal to or more than 20 deep CMBs. Assignment of lobar CMB severity: $0=$ absence of lobar CMB, 1 = 1-4 lobar CMBs, $2=5-9$ lobar CMBs, 3 = 10-19 lobar CMBs, $4=$ equal to or more than 20 lobar CMBs.

(cumulative CSVD score or PWMH + lobar CMB severity) for stroke recurrence was not significantly different from that of an individual marker (Supplementary Table 4).

\section{CSVD Burden and Time-Varying Survival}

The median value of the cumulative CSVD score was 2 (1-3); so, the prespecified high cumulative CSVD burden was defined as the cumulative CSVD score $\geq 2$. The Cox proportional hazards regression analysis showed that only $\mathrm{CMBs} \geq 10$ was significantly related to mortality (adjusted HR 2.669, 95\% CI 1.248-5.707; $p=0.011)$ after adjusting for age, sex, hypertension, cardiac disease, GCS, albumin, and any complication. Additionally, a higher combined CSVD burden was associated with a decreased survival rate (adjusted HR 3.140, 95\% CI 1.066-9.250, $p=0.038$ 
for cumulative CSVD score $\geq 2$; adjusted HR 1.457, 95\% CI $1.049-2.024, p=0.025$ for $\mathrm{CMB} \geq 10+\mathrm{PWMH}$ ) (Figure 2). We found no statistically significant differences in survival, stratified by lacune, PWMH, DWMH, or EPVS.

\section{DISCUSSION}

The key findings of this study are that severe PWMH and CMB were independent predictors of poor functional outcome. There was a substantial risk of stroke recurrence in patients with greater PWMH or lobar CMB burden in patients with ICH. As expected, cumulative CSVD burden altered the occurrence risk of each of the three prespecified outcomes in long-term follow-up, mainly because of the comprehensive effect of $\mathrm{WMH}$ and $\mathrm{CMB}$. The predictive value of the combined score $(\mathrm{CMB} \geq 10+\mathrm{PWMH})$ for poor functional outcome performed better than that of the individual marker of $\mathrm{CMB} \geq 10$ or $\mathrm{CMB} \geq 5$.

In our present study, severe PWMH were significantly associated with poor functional outcome and stroke recurrence, independent of age and other potential confounders. Patients with a high PWMH score were older ( $p<0.001$, ANOVA) and more likely to have hypertension $(p<0.05$, the chi-squared test) than those with a low PWMH score. Severe PWMH was significantly associated with the presence of mixed-location $\mathrm{ICH}$ ( $p<0.001$, the chi-squared test) in this study. As shown in the previous literature, mixed ICHs were more likely to have

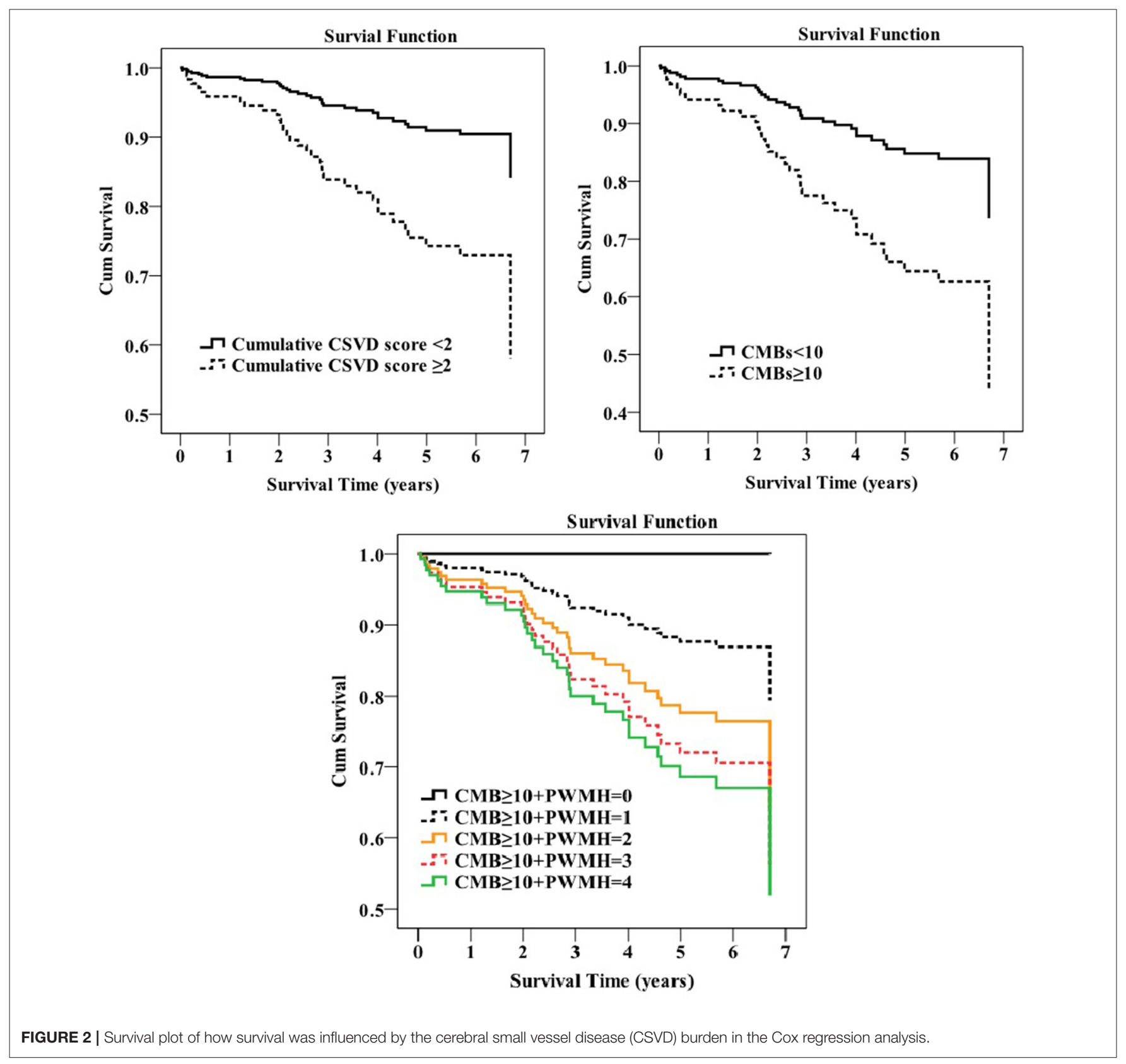


pronounced classic vascular risk factor burden (hypertension, diabetes mellitus, and creatinine) and high $\mathrm{WMH}$ volume compared to HA-ICH or CAA-ICH ( $p<0.001$ ) (Pasi et al., 2018), suggesting that the burden of classic vascular risk factor could be a critical player in the development of a severe arteriopathy in the brain. The mechanism underlying why PWMH, but not DWMH, was associated with a poor outcome is not clear. Perhaps, the vascular architecture in periventricular white matter is more susceptible to damage than that in other areas (Pantoni and Garcia, 1997). Anatomically, there is a high density of long association fibers connecting the cortex with distant brain territories and subcortical nuclei, while the subcortical region has a high density of short-looped U-fibers, which connect adjacent regions (Filley, 1998; De Groot et al., 2002). Lesions affecting long association fibers may have more influence on function than lesions affecting connectivity between neighboring brain areas because of possibly reserve mechanisms of the latter (De Groot et al., 2002).

The strong association of $\mathrm{CMB}$ with the long-term poor functional outcome is a novel observation. Lioutas et al. (2018) reported a significant detrimental effect of CMB on 90-days functional independence ( $\mathrm{mRS} \leq 2)$ (Lioutas et al., 2018). Our study extended the follow-up to a median of 5 years, verifying the detrimental effect of $\mathrm{CMB}$ on long-term prognosis, and further found that PWMH, not DWMH, was closely associated with a poor outcome and stroke recurrence.

The association between the lobar CMB severity and the stroke recurrence is expected. Histopathologically, $\mathrm{CMB}$ corresponds with the focal deposition of hemosiderin surrounding small vessels (Greenberg et al., 2009). Those lobar CMBs, which might be due to CAA (Greenberg et al., 2009), are correlated to a greater beta-amyloid burden than deep CMBs (Tsai et al., 2017). A previous study showed that the lobar $\mathrm{CMB}$ number was associated with the recurrence of lobar $\mathrm{ICH}$ during a median follow-up of 34.3 months (Biffi et al., 2010). A pooled analysis of 10 studies also found that multiple baseline $\mathrm{CMBs}$ were associated with the recurrence during follow-up (Charidimou et al., 2017).

\section{Strength and Limitations}

First, we used 3-T SWI to detect CMB. Second, although recurrence was more common in the lobar $\mathrm{ICH}$ than in the deep ICH as per the previous systematic reviews (Bailey et al., 2001; Poon et al., 2014), probably because of the type and severity of the underlying microangiopathies (Pantoni, 2010), few studies have systematically addressed the phenotype of the underlying small vessel disease type (Bailey et al., 2001; Poon et al., 2014). In our present study, we used MRI which is the most useful and noninvasive method to phenotype microangiopathies (Charidimou et al., 2017) to directly control the association between CSVD burden and stroke recurrence. Third, our follow-up was relatively long. As reported in a recent study, management in the stroke unit or neurointensive care unit might influence stroke prognosis (Pilato et al., 2020). In our study, we included eligible patients who were admitted to either the Department of Neurosurgery or the Department of Neurology in West China Hospital; both departments are high-volume stroke centers, as well as diagnosis and treatment centers for complicated and severe neurological diseases in Southwest China. All the included patients were treated according to current guidelines.

However, this present study has several limitations. There might have been selection bias because not all patients had an MRI; thus, the findings could only be generalized to patients with ICH who undergo MRI in clinical practice. Since the included sample size was relatively small, we were unable to test the influence of individual $\mathrm{CMB}$ markers and the cumulative CSVD score on this long-term prognosis in each of the ICH subtypes. Finally, we followed after discharge, and stroke recurrence was confirmed by asking patients or their family members, so the possibility of recall bias could not be excluded. In future, large studies are needed to verify our results.

\section{CONCLUSION}

We concluded that the increasing burden of PWMH, CMB, and combined CSVD burden exerts important influence on poor functional outcome and the stroke recurrence in patients with $\mathrm{ICH}$. CMBs $\geq 10$ and the combined CSVD burden are significantly related to time-varying survival. Our findings are important to stratify patients with $\mathrm{ICH}$ who are more prone to poor clinical outcomes. Our data demonstrated that MRI is valuable in assessing prognosis in patients with primary $\mathrm{ICH}$.

\section{DATA AVAILABILITY STATEMENT}

The raw data supporting the conclusions of this article will be made available by the authors, without undue reservation.

\section{ETHICS STATEMENT}

The studies involving human participants were reviewed and approved by Ethics Committee on Biomedical Research, West China Hospital of Sichuan University. The patients/participants provided their written informed consent to participate in this study.

\section{AUTHOR CONTRIBUTIONS}

MX and ML designed the study. MX, BL, DZ, YC, and QW enrolled and followed subjects. MX and YC reviewed clinical head CTs and MRIs for radiologic grading. MX carried out data analysis and wrote the manuscript. Important data analysis suggestions and manuscript revisions were made by BW, ML, ShuZ, and ShiZ. All authors contributed to the article and approved the submitted version.

\section{FUNDING}

This study was supported by the National Key R\&D Program of China, Ministry of Science and Technology of China (2016YFC1300500-505), the National Natural Science 
Foundation of China (Grant No. 82001250), the China Postdoctoral Science Foundation (Grant No. 2020M683322), and the 1.3.5 Project for Disciplines of Excellence, West China Hospital, Sichuan University (Grant No. ZYGD 18009).

\section{REFERENCES}

Bailey, R. D., Hart, R. G., Benavente, O., and Pearce, L. A. (2001). Recurrent brain hemorrhage is more frequent than ischemic stroke after intracranial hemorrhage. Neurology 56, 773-777. doi: 10.1212/WNL.56. 6.773

Biffi, A., Halpin, A., Towfighi, A., Gilson, A., Busl, K., Rost, N., et al. (2010). Aspirin and recurrent intracerebral hemorrhage in cerebral amyloid angiopathy. Neurology 75, 693-698. doi: 10.1212/WNL.0b013e3181e ee $40 \mathrm{f}$

Charidimou, A., Boulouis, G., Haley, K., Auriel, E., van Etten, E. S., Fotiadis, P., et al. (2016). White matter hyperintensity patterns in cerebral amyloid angiopathy and hypertensive arteriopathy. Neurology 86, 505-511. doi: 10.1212/WNL.0000000000002362

Charidimou, A., Imaizumi, T., Moulin, S., Biffi, A., Samarasekera, N., Yakushiji, Y., et al. (2017). Brain hemorrhage recurrence, small vessel disease type, and cerebral microbleeds: a meta-analysis. Neurology 89, 820-829. doi: 10.1212/WNL.0000000000004259

De Groot, J. C., De Leeuw, F. E., Oudkerk, M., Van Gijn, J., Hofman, A., Jolles, J., et al. (2002). Periventricular cerebral white matter lesions predict rate of cognitive decline. Ann. Neurol. 52, 335-341. doi: 10.1002/ana.10294

Dellinger, R. P., Levy, M. M., Rhodes, A., Annane, D., Gerlach, H., Opal, S. M., et al. (2013). Surviving sepsis campaign: international guidelines for management of severe sepsis and septic shock: 2012. Crit. Care Med. 41, 580-637. doi: 10.1097/CCM.0b013e31827e83af

DeLong, E. R., DeLong, D. M., and Clarke-Pearson, D. L. (1988). Comparing the areas under two or more correlated receiver operating characteristic curves: a nonparametric approach. Biometrics 44, 837-845. doi: 10.2307/2531595

Fazekas, F., Chawluk, J. B., Alavi, A., Hurtig, H. I., and Zimmerman, R. A. (1987). MR signal abnormalities at $1.5 \mathrm{~T}$ in Alzheimer's dementia and normal aging. AJR 149, 351-356. doi: 10.2214/ajr.149.2.351

Filley, C. M. (1998). The behavioral neurology of cerebral white matter. Neurology 50, 1535-1540. doi: 10.1212/WNL.50.6.1535

Greenberg, S. M., Vernooij, M. W., Cordonnier, C., Viswanathan, A., Al-Shahi Salman, R., Warach, S., et al. (2009). Cerebral microbleeds: a guide to detection and interpretation. Lancet Neurol. 8, 165-174. doi: 10.1016/S1474-4422(09)70013-4

Gregório, T., Pipa, S., Cavaleiro, P., Atanásio, G., Albuquerque, I., Chaves, P. C., et al. (2018). Prognostic models for intracerebral hemorrhage: systematic review and meta-analysis. BMC Med. Res. Methodol. 18:145. doi: 10.1186/s12874-018-0613-8

Hankey, G. J. (2017). Stroke. Lancet 389, 641-654. doi: 10.1016/S0140-6736(16)30962-X

Indredavik, B., Rohweder, G., Naalsund, E., and Lydersen, S. (2008). Medical complications in a comprehensive stroke unit and an early supported discharge service. Stroke 39, 414-420. doi: 10.1161/STROKEAHA.107. 489294

Klarenbeek, P., van Oostenbrugge, R. J., Rouhl, R. P., Knottnerus, I. L., and Staals, J. (2013). Ambulatory blood pressure in patients with lacunar stroke: association with total MRI burden of cerebral small vessel disease. Stroke 44, 2995-2999. doi: 10.1161/STROKEAHA.113.002545

Kothari, R. U., Brott, T., Broderick, J. P., Barsan, W. G., Sauerbeck, L. R., Zuccarello, M., et al. (1996). The ABCs of measuring intracerebral hemorrhage volumes. Stroke 27, 1304-1305. doi: 10.1161/01.STR.27.8.1304

Langhorne, P., Stott, D. J., Robertson, L., MacDonald, J., Jones, L., McAlpine, C., et al. (2000). Medical complications after stroke: a multicenter study. Stroke 31, 1223-1229. doi: 10.1161/01.STR.31.6.1223

Li, J., Zhang, P., Wu, S., Wang, Y., Zhou, J., Yi, X., et al. (2019). Strokerelated complications in large hemisphere infarction: incidence and influence

\section{SUPPLEMENTARY MATERIAL}

The Supplementary Material for this article can be found online at: https://www.frontiersin.org/articles/10.3389/fnagi. 2021.628271/full\#supplementary-material

on unfavorable outcome. Ther. Adv. Neurol. Disord. 12:1756286419873264 doi: $10.1177 / 1756286419873264$

Linn, J., Halpin, A., Demaerel, P., Ruhland, J., Giese, A. D., Dichgans, M., et al. (2010). Prevalence of superficial siderosis in patients with cerebral amyloid angiopathy. Neurology 74, 1346-1350. doi: 10.1212/WNL.0b013e3181dad605

Lioutas, V. A., Wu, B., Norton, C., Helenius, J., Modak, J., and Selim, M. (2018). Cerebral small vessel disease burden and functional and radiographic outcomes in intracerebral hemorrhage. J. Neurol. 265, 2803-2814. doi: 10.1007/s00415-018-9059-5

Pantoni, L. (2010). Cerebral small vessel disease: from pathogenesis and clinical characteristics to therapeutic challenges. Lancet Neurol. 9, 689-701. doi: 10.1016/S1474-4422(10)70104-6

Pantoni, L., and Garcia, J. H. (1997). Pathogenesis of leukoaraiosis: a review. Stroke 28, 652-659. doi: 10.1161/01.STR.28.3.652

Pasi, M., Charidimou, A., Boulouis, G., Auriel, E., Ayres, A., Schwab, K. M., et al. (2018). Mixed-location cerebral hemorrhage/microbleeds: Underlying microangiopathy and recurrence risk. Neurology 90:e119-e26. doi: 10.1212/WNL.0000000000004797

Pilato, F., Silva, S., Valente, I., Distefano, M., Broccolini, A., Brunetti, V., et al. (2020). Predicting factors of functional outcome in patients with acute ischemic stroke admitted to neuro-intensive care unit-a prospective cohort study. Brain Sci. 10:911. doi: 10.3390/brainsci10120911

Poon, M. T., Fonville, A. F., and Al-Shahi Salman, R. (2014). Long-term prognosis after intracerebral haemorrhage: systematic review and meta-analysis. J. Neurol. Neurosurg. Psychiatr. 85, 660-667. doi: 10.1136/jnnp-2013-306476

Song, T. J., Kim, J., Song, D., Yoo, J., Lee, H. S., Kim, Y. J., et al. (2017). Total cerebral small-vessel disease score is associated with mortality during follow-up after acute ischemic stroke. J. Clin. Neurol. 13, 187-195. doi: 10.3988/jcn.2017.13.2.187

Staals, J., Makin, S. D., Doubal, F. N., Dennis, M. S., and Wardlaw, J. M. (2014). Stroke subtype, vascular risk factors, and total MRI brain small-vessel disease burden. Neurology 83, 1228-1234. doi: 10.1212/WNL.0000000000000837

Sykora, M., Herweh, C., and Steiner, T. (2017). The association between leukoaraiosis and poor outcome in intracerebral hemorrhage is not mediated by hematoma growth. J. Stroke Cerebrovasc. Dis. 26, 1328-1333. doi: 10.1016/j.jstrokecerebrovasdis.2017.02.003

Tsai, H. H., Tsai, L. K., Chen, Y. F., Tang, S. C., Lee, B. C., Yen, R. F., et al. (2017). Correlation of cerebral microbleed distribution to amyloid burden in patients with primary intracerebral hemorrhage. Sci. Rep. 7:44715. doi: $10.1038 /$ srep 44715

Uniken Venema, S. M., Marini, S., Lena, U. K., Morotti, A., Jessel, M., Moomaw, C. J., et al. (2019). Impact of cerebral small vessel disease on functional recovery after intracerebral hemorrhage. Stroke 50, 2722-2728. doi: 10.1161/STROKEAHA.119.025061

Valenti, R., Del Bene, A., Poggesi, A., Ginestroni, A., Salvadori, E., Pracucci, G., et al. (2016). Cerebral microbleeds in patients with mild cognitive impairment and small vessel disease: The Vascular Mild Cognitive Impairment (VMCI)-Tuscany study. J. Neurol. Sci. 368, 195-202. doi: 10.1016/j.jns.2016. 07.018

Wardlaw, J. M., Smith, E. E., Biessels, G. J., Cordonnier, C., Fazekas, F., Frayne, R., et al. (2013). Neuroimaging standards for research into small vessel disease and its contribution to ageing and neurodegeneration. Lancet Neurol. 12, 822-838. doi: 10.1016/S1474-4422(13)70 124-8

Xu, M., Cheng, Y., Song, Q., Yuan, R., Zhang, S., Hao, Z., et al. (2019). Total burden of cerebral small vessel disease in recurrent ich versus first-ever ICH. Aging Dis 10, 570-577. doi: 10.14336/AD.2018.0804

Xu, M., Zhang, S., Liu, J., Luo, H., Wu, S., Cheng, Y., et al. (2018). Kidney dysfunction is associated with a high burden of cerebral 
small vessel disease in primary intracerebral hemorrhage. Curr. Neurovasc. Res. 15, 39-46. doi: 10.2174/15672026156661803260 94728

Conflict of Interest: The authors declare that the research was conducted in the absence of any commercial or financial relationships that could be construed as a potential conflict of interest.
Copyright (C) $2021 \mathrm{Xu}, \mathrm{Li}$, Zhong, Cheng, Wu, Zhang, Zhang, Wu and Liu. This is an open-access article distributed under the terms of the Creative Commons Attribution License (CC BY). The use, distribution or reproduction in other forums is permitted, provided the original author(s) and the copyright owner(s) are credited and that the original publication in this journal is cited, in accordance with accepted academic practice. No use, distribution or reproduction is permitted which does not comply with these terms. 